OPEN ACCESS

Edited by:

Djamel Drider,

Lille University of Science

and Technology, France

Reviewed by:

Olga Soutourina,

UMR 9198 Institut de Biologie Intégrative de la Cellule (I2BC), France

Veronique Delcenserie,

University of Liège, Belgium

*Correspondence:

Yanxia Wei

weiyx2007@aliyun.com

Kuiyang Zheng

zky02@163.com

Renxian Tang

tangrenxian-t@163.com

tThese authors have contributed equally to this work.

Specialty section: This article was submitted to

Food Microbiology,

a section of the journal

Frontiers in Microbiology

Received: 24 December 2017

Accepted: 18 April 2018

Published: 08 May 2018

Citation:

Wei Y, Yang F, Wu Q, Gao J, Liu W, Liu C, Guo X, Suwal S, Kou Y, Zhang $B$, Wang $Y$, Zheng $K$ and Tang $R$ (2018) Protective Effects of Bifidobacterial Strains Against

Toxigenic Clostridium difficile.

Front. Microbiol. 9:888.

doi: 10.3389/fmicb.2018.00888

\section{Protective Effects of Bifidobacterial Strains Against Toxigenic Clostridium difficile}

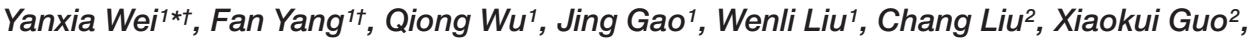 \\ Sharmila Suwal' ${ }^{1}$, Yanbo Kou ${ }^{1}$, Bo Zhang ${ }^{1}$, Yugang Wang ${ }^{1}$, Kuiyang Zheng ${ }^{1 *}$ and \\ Renxian Tang ${ }^{1 *}$
}

\begin{abstract}
1 Jiangsu Key Laboratory of Immunity and Metabolism, Department of Pathogenic Biology and Immunology, Laboratory of Infection and Immunity, Xuzhou Medical University, Xuzhou, China, ${ }^{2}$ Department of Microbiology and Immunology, Shanghai Jiao Tong University School of Medicine, Shanghai, China
\end{abstract}

Probiotics might offer an attractive alternative to prevent and control Clostridium difficile (C. difficile) infection (CDI). Limited information is available on the ability of commercially used bifidobacterial strains to inhibit $C$. difficile. This study examined the anti-clostridial effects of Bifidobacterium longum JDM301, a widely used commercial probiotic strain in China, in vitro and in vivo. In vitro evaluation revealed a significant reduction in $C$. difficile counts when JDM301 was co-cultured with $C$. difficile, which was correlated with the significant decrease in clostridial toxin titres (TcdA and TcdB). Furthermore, the cellfree culture supernatants (CFS) of JDM301 inhibited C. difficile growth and degraded TcdA and TcdB. Notably, the results showed that acid pH promoted the degradation of TcdA by CFS from JDM301. Furthermore, comparative studies among $10 \mathrm{~B}$. longum strains were performed, which showed that the inhibitory effect of CFS from JDM301 was similar with the other 8 B. longum strains and higher than strain BLY1. However, when it was neutralized, the significant different was lost. When present together, it was suggested that the acid $\mathrm{pH}$ induced by probiotics not only played important roles in the growth inhibition against $C$. difficile resulting in the reduction of toxins titres, but also directly promoted the degradation of clostridial toxin. In vivo studies proved that JDM301 partially relieved damage to tissues caused by $C$. difficile and also decreased the number of $C$. difficile and toxin levels. In summary, our results demonstrated that the commercial strain, JDM301 could be considered a probiotic able to exert anti-toxin capability and most of the CFS from Bifidobacterium were able to inhibit the growth of C. difficile, depending on acid $\mathrm{pH}$. These results highlighted a potential that JDM301 could be helpful in preventing CDI and that most of the bifidobacterial strains could (at least partially) exert protective effects by reducing toxin titres through growth inhibition against toxigenic $C$. difficile.

Keywords: Bifidobacterium, Clostridium difficile, probiotics, protective effect, Toxin A, Toxin B, degradation

\section{INTRODUCTION}

Clostridium difficile (C. difficile) is an obligate anaerobic gram-positive spore-forming bacillus. C. difficile is responsible for $15-39 \%$ of all antibiotic-associated diarrhea cases and the cause of pseudomembranous colitis in human (Badger et al., 2012). The pseudomembranous colitis and toxic megacolon led by severe infections of $C$. difficile are potentially fatal (Rupnik et al., 2009). 
The occurrence of $C$. difficile-associated infections is increasing in recent years (Louh et al., 2017). Toxin A (TcdA) and toxin B (TcdB) from $C$. difficile are its main virulence factors. The two genes $t c d A$ and $t c d B$, encoding TcdA and $T c d B$, respectively, were located at pathogenicity locus (Mani and Dupuy, 2001; Carter et al., 2011). The mechanisms regulating the production of the toxins remained largely unknown. Darkoh et al. (2015) showed that $C$. difficile toxin synthesis could be regulated by quorumsignaling system. As intracellular glycosyltransferases, TcdA and TcdB could modify the Ras superfamily of small GTPase resulting in intracellular changes, such as F-actin condensation and cell apoptosis of intestinal epithelial cells, which would lead to the damage of intestinal barrier integrity, inflammatory response and fluid accumulation in gut (Kim et al., 2007; Carter et al., 2015; Leslie et al., 2015). C. difficile infection (CDI) mouse exhibited a significant increase of IL- 6 production, a marker of acute inflammation (Kim et al., 2007; Kang et al., 2011). The production of another inflammatory cytokine, TNF- $\alpha$ also increased significantly in gut of CDI mice (Li et al., 2012).

The alteration of gut flora caused by antibiotic treatment allowed the colonization and growth of C. difficile (Britton and Young, 2011). Several antibiotics were involved in the occurrence of antibiotic-associated diarrhea. The rate of diarrhea varied according to the antibiotic used. The rate associated with cefixime or amoxicillin-clavulanate was highest, which was $15-25 \%$ or $10-25 \%$, respectively (Bartlett, 2002). The infection of $C$. difficile was responsible for the majority of cases of colitis associated with antibiotic therapy (Bartlett, 2002), although it only accounted for 15-39\% of all antibiotic-associated diarrhea cases (Badger et al., 2012). It has been shown that the indigenous microflora in gut plays a protective role for enteric infections, particularly for CDI (Juszczuk et al., 2017; Petrosillo, 2018). There was markedly disrupted intestinal microbiota in patients with CDI, particularly people with recurrent CDI (Evans and Johnson, 2015). A progressive loss of diversity and dropout of major bacterial groups were observed in CDI. The diversity of gut microbiota from patients who developed CDI after antibiotic treatment decreased compared with patients who did not develop CDI (Chang et al., 2008). The prevention and treatment of CDI has remained a challenge. So far, there are a limited number of antibiotics available for the treatment of CDI. Up to $20 \%$ of patients treated by antibiotic administration will experience a recurrence of CDI following treatment (Petrosillo, 2018). CDI has been treated by vancomycin and/or metronidazole (Kociolek and Gerding, 2016). However, with the emergence of hypervirulent antibiotic-resistant strains, the incidence of $C$. difficile-associated diarrhea and intestinal inflammatory disease has increased significantly, becoming a public health problem (McDonald et al., 2005). Furthermore, standard treatment by antibiotics for CDI results in disruptive effect on the colonic microbiota (Kociolek and Gerding, 2016). The altered indigenous microbiota is a principle risk factor of CDI, since it allows ingested spores of $C$. difficile to germinate and proliferate. An environment in which cholate levels are markedly higher than other primary and secondary bile acids would promote germination of $C$. difficile spores (Britton and Young, 2011). Administration of antibiotics increases the level of cholate by disrupting the normal microbiota in gut (Giel et al., 2010). As stated by (Kociolek and Gerding, 2016; Petrosillo, 2018), the standard antibiotic treatment for CDI (metronidazole and vancomycin), was limited by their broad spectrum and further disturbance of the intestinal flora. In the past few years, there has been a renewed recognition in CDI that the disease is more common and more resistant to standard treatment (Evans and Johnson, 2015; Geeraerts et al., 2015). Compared with antibiotics, a major advantage of probiotics is that it is less possible to increase the incidence of antibiotic resistance (Rolfe, 2000; Evans and Johnson, 2015). Due to the limitations of antibiotic treatment, there is an urgent need to develop alternative strategies for prevention and treatment of CDI. Fecal microbiota transplantation (FMT) and the approach using probiotics and/or prebiotics are promising methods (Hudson et al., 2017). Probiotics and/or prebiotics seem to be safer than FMT because the latter involves a more complex extract prepared from homogenized stool sample (Anand et al., 2017). Probiotics can protect the host against pathogens by restoring the complex balance of the indigenous microbiota in gut, modulation of immune system and inhibition of the growth of pathogen, which can limit the need for additional antibiotic treatment (Parkes et al., 2009; Kumar et al., 2016).

Several studies have demonstrated that probiotic strains appear to reduce the incidence of CDI, in which Lactobacillus rhamnosus and Saccharomyces boulardii have been found to be associated with a significant reduction in antibiotic associated diarrhea. A few studies have showed the inhibition of $C$. difficile by bifidobacterial strains with different levels of success (Goldenberg et al., 2017; Mills et al., 2018). The inhibitory activity of $B$. animalis sp. lactis against $C$. difficile in vitro was reported (Schoster et al., 2013). Four strains belonging to $B$. longum were able to reduce the toxic effect of $C$. difficile upon HT29 monolayers (Valdes-Varela et al., 2016). Trejo et al. (2013) reported that the B. bifidum strain CIDCA 5310 could antagonize the virulence of $C$. difficile in vivo. Yun et al. (2017) showed beneficial effect of B. longum ATCC 15707 on survival rate of CDI in mice. However, the related studies remain scarce in vitro and in vivo, especially for commercially used strains (Parkes et al., 2009; Evans and Johnson, 2015). Probiotics may prevent or treat CDI through multiple possible mechanisms. Lactic acid and other organic acids have been considered to play important roles in antibacterial activity of bifidobacterial strains against C. difficile (Schoster et al., 2013). Compounds secreted by Bacillus clausii could inhibit the cytotoxic effects induced by C. difficile (Ripert et al., 2016). L. plantarum and xylitol inhibited in vitro germination of $C$. difficile spores (Ratsep et al., 2017). L. reuteri could convert glycerol to the broad-spectrum antimicrobial compound reuterin that directly inhibited the growth of C. difficile (Spinler et al., 2017). Two strains from Lactobacillus could suppress $C$. difficile-induced IL-8 production from HT-29 cells (Boonma et al., 2014). To date, studies related to the role of bifidobacteria in modulating the immune response against CDI are limited, especially for widely used bifidobacterial strain in vivo. Additionally, the in vivo activities of bifidobacteria against $C$. difficile were normally associated 
with other probiotics in these previous studies (Mills et al., 2018).

Bifidobacterium is a dominant genus which is beneficial to humans and considered as a model probiotic bacterium. $B$. longum is a gram-positive bacterium commonly found in the guts of healthy humans. Some strains of $B$. longum can prevent gastroenteritis or colitis induced by rotavirus or 2,4,6trinitrobenzenesulfonic acid (Lee et al., 2009; Munoz et al., 2011). Due to the benefits associated with B. longum, it is often added to a large array of probiotic foods and dietary supplements. $B$. longum is the most common bifidobacterial species utilized as a probiotic in commercial products (Sheu et al., 2010). However, limited information is available on the antagonistic activity of commercial bifidobacteria against $C$. difficile, especially in vivo.

In this study, the impact on toxin production and cell growth of $C$. difficile by $B$. longum JDM301 were assessed and the anti-toxin (TcdA and TcdB) capability of JDM301 supernatant was investigated in vitro. Furthermore, the efficacy of JDM301 to prevent CDI was evaluated in a mouse model. In addition, comparative studies among bifidobacterial strains were performed to reveal the difference among the inhibitory effects against $C$. difficile growth by cell-free culture supernatants (CFS) from these symbiotic bacteria.

\section{MATERIALS AND METHODS}

\section{Bacterial Strains and Growth Conditions}

Total of 25 strains of Bifidobacterium and 15 strains of other bacteria were used in this study (Supplementary Table S1). Nine strains were isolated from commercial probiotic products and the others were isolated from healthy Chinese infants (aged from 1 to 24 months). Fresh stool samples were collected from healthy infants. The fresh samples were homogenized in sterile phosphate saline buffer (PBS, $0.144 \mathrm{~g} \mathrm{KH}_{2} \mathrm{PO}_{4} / \mathrm{l}, 9 \mathrm{~g} \mathrm{NaCl} / \mathrm{l}, 0.795 \mathrm{~g}$ $\mathrm{Na}_{2} \mathrm{HPO}_{4} / \mathrm{l}, \mathrm{pH}$ 7.5) supplemented with $0.05 \%$ (w/v) L-cysteine$\mathrm{HCl}$ and filtered through two layers of sterile gauze sponges to obtain final human fecal inocula. These inocula were cultured to isolate strains belonging to Bifidobacterium or Lactobacillus genus. The study was approved by the ethics committee of Xuzhou Medical University and informed consent was obtained from all parents. These strains included B. bifidum $(n=5)$, B. longum $(n=10), B$. animalis $(n=5), B$. pseudocatenulatum $(n=3)$, B. breve $(n=2)$, L. casei $(n=3)$, L. plantarum $(n=5)$, and one each of L. crispatus, L. fermentum, L. rhamnosus, L. acidophilus, L. gasseri, L. delbrueckii, Enterococcus avium and Streptococcus pasteurianus. All the strains were identified using a sequence analysis of their 16S rRNA genes and cultured in MRS (Difco) supplemented with $0.05 \%(\mathrm{w} / \mathrm{v}) \mathrm{L}$-cysteine- $\mathrm{HCl}$. Among them, B. longum JDM301 (JDM301), was a widely used Chinese commercial strain and its complete genome sequence was determined (Wei et al., 2010). Anti-clostridial activity was measured on the reference strains C. difficile strain ATCC 43255 and ATCC 9689. C. difficile ATCC 43255 and ATCC 9689 were purchased from ATCC (American Type Culture Collection, Manassas, VA, United States). The strain ATCC 9689 and ATCC 43255 can produce both TcdA and TcdB (Babakhani et al., 2013;
Valdes-Varela et al., 2016). Other strains were cultured in brainheart infusion (BHI) medium (Oxoid) supplemented with $0.05 \%$ $(\mathrm{w} / \mathrm{v}) \mathrm{L}$-cysteine-HCl when they or their supernatants were cocultured or incubated with C. difficile. C. difficile strains were also cultured in BHI (Oxoid). Cultures were incubated at $37^{\circ} \mathrm{C}$ in an anaerobic workstation (Don Whitley Scientific).

\section{Co-culture of B. longum JDM301 and C. difficile}

For co-culture of $C$. difficile with B. longum JDM301, C. difficile and $B$. longum JDM301 in exponential growth phase were harvested by centrifugation and washed three times with PBS, respectively. The pellets were resuspended in BHI-cys/agar 0.05\% $(\mathrm{w} / \mathrm{v})$ at different inoculation ratios (B. longum: C. difficile). C. difficile ATCC 43255 or ATCC 9689 was used to co-culture with B. longum JDM301 in BHI-cys/agar 0.05\% (w/v) for $24 \mathrm{~h}$ at $37^{\circ} \mathrm{C}$ under anaerobic conditions, respectively. Then, the counts of $C$. difficile were determined by plating serial dilutions of the cultures on BHI-cys/agar $0.05 \%(\mathrm{w} / \mathrm{v})$. Plates were incubated at $37^{\circ} \mathrm{C}$ for $48 \mathrm{~h}$ in anaerobic conditions.

\section{Growth of C. difficile in the Supernatants of Different Bifidobacterium Strains and Other Symbiotic Bacteria}

The inhibitory effects of CFS from 40 strains of Bifidobacterium $(n=24)$, Lactobacillus $(n=13)$, Enterococcus $(n=1)$, and Streptococcus $(n=1)$ were tested as described previously (MunozQuezada et al., 2013) with a few modifications. The strains were grown anaerobically in BHI medium supplemented with $0.05 \%(w / v)$ L-cysteine-HCl. Strain cultures were adjusted to an optical density of 0.5 at $600 \mathrm{~nm}\left(\mathrm{OD}_{600}\right)$. Then cultures of the symbiotic bacteria were transferred into fresh BHI-cys $0.05 \%(\mathrm{w} / \mathrm{v})$ using a $10 \%$ inoculum and incubated at $37^{\circ} \mathrm{C}$ for $24 \mathrm{~h}$ anaerobically. After $24 \mathrm{~h}$, the supernatants were obtained by centrifugation at $13,400 \mathrm{rpm}$ for $5 \mathrm{~min}$ and divided into two aliquots, one of which was neutralized to $\mathrm{pH} 7.0$ using $\mathrm{NaOH}$. Both aliquots were filter-sterilized using $0.22 \mu \mathrm{m}$ filters and stored at $-80^{\circ} \mathrm{C}$ until further use. C. difficile cultures were adjusted to 0.5 at $\mathrm{OD}_{600}$ and $300 \mu \mathrm{l}$ of the cultures were added to $3 \mathrm{ml} \mathrm{BHI-cys} 0.05 \%(\mathrm{w} / \mathrm{v})$ or the CFS of different symbiotic bacteria with or without $\mathrm{pH}$ neutralization, respectively. Growth was measured spectrophotometrically at $24 \mathrm{~h}$ after anaerobic incubation at $37^{\circ} \mathrm{C}$. The growth of C. difficile cultured in BHI-cys $0.05 \%(\mathrm{w} / \mathrm{v})$ was defined as $100 \%$. The percentage of growth was calculated by comparing the final $\mathrm{OD}_{600}$ obtained with C. difficile cultured in CFS of different symbiotic bacteria with those of the corresponding control samples [C. difficile cultured in BHI-cys $0.05 \%(w / v)]$.

\section{Incubation of C. difficile Supernatant With JDM301 Supernatant}

JDM301 and C. difficile ATCC 43255 were cultured anaerobically at $37^{\circ} \mathrm{C}$ for $24 \mathrm{~h}$, respectively. After $24 \mathrm{~h}$, the $\mathrm{pH}$ in supernatant obtained from JDM301 in late-log phase, was approximately 4.9. The cultures of JDM301 or C. difficile ATCC 43255 were centrifuged at 13,400 rpm for $5 \mathrm{~min}$. The supernatant from 
JDM301 was divided into two aliquots, one of which was neutralized to $\mathrm{pH} 7.0$ using $\mathrm{NaOH}$. Then both aliquots and the supernatant from ATCC 43255 were all filter-sterilized using $0.22 \mu \mathrm{m}$ filter. For incubation of $C$. difficile supernatant with JDM301 supernatant, the CFS of $C$. difficile was incubated with CFS of B. longum JDM301 ( $\mathrm{pH}=4.9$ ) or neutralized JDM301-CFS $(\mathrm{pH}=7.0)$ for $48 \mathrm{~h}$ at $37^{\circ} \mathrm{C}$ under anaerobic conditions. Then, toxins (TcdA and TcdB) in these co-incubated new supernatants were detected by Dot-blot assay.

\section{Toxin Assay}

The production of TcdA and TcdB was determined by commercially available ELISA kits (Senbeijia) or Dot-blot assay. The commercial kits were used and standard curves were obtained for ELISA as recommended by the manufacturer. Dot-blot assay was performed as reported previously with a few modifications (Bolla et al., 2013). Total of $5 \mu \mathrm{l}$ samples were spotted onto nitrocellulose membranes. After drying for $30 \mathrm{~min}$ at room temperature, strips were blocked. Afterwards, TcdA and TcdB were analyzed by immunoblotting using antiTcdA monoclonal antibodies (Abcam) or anti-TcdB monoclonal antibodies (Abcam). A horseradish peroxidase-labeled goatanti-mouse IgG antibody was used as a secondary antibody. Chemiluminescence signals were visualized with an enhanced chemiluminescence (ECL) reagent (Thermo Scientific) and exposed on film.

\section{Quantification of C. difficile in Cecal Contents by Quantitative PCR (qPCR)}

The samples of cecal content were weighted before DNA extraction. The DNA of cecal content was extracted under the protocol provided in the QIAamp DNA Stool Mini Kit (QIAGEN). C. difficile DNA from extracted DNA of cecal content was analyzed by qPCR. The $t c d B$ gene was used as a specific marker of $C$. difficile. Primers targeting $t c d B$ reported previously (Babakhani et al., 2013) were used for quantification of $C$. difficile DNA. The sequence of $t c d B$ forward primer was oLB141 (GGCAAATGTAAGATTTCGTACTCA) and the sequence of $t c d B$ reverse primer was oLB142 (TCGACTACAGTATTCTCTGAC). The size of qPCR product was 96 bp. $t c d B$ was amplified and cloned into pEASY-T1 cloning vector using pEASY-T1 cloning kit (TransGen Biotech). The T-vector carrying $t c d B$ was created and used as template to generate standard curve in this study. The $\mathrm{qPCR}$ reaction was performed with the FastStart Universal SYBR Green Master (Roche) using the following conditions: $95^{\circ} \mathrm{C}$ for $10 \mathrm{~min}$, followed by 40 cycles at $95^{\circ} \mathrm{C}$ for $15 \mathrm{~s}$ and $60^{\circ} \mathrm{C}$ for $1 \mathrm{~min}$. The standard curve was generated with the plasmid carrying the partial $t c d B$ gene. Copy numbers of $C$. difficile in cecal contents were extrapolated from the standard curve.

\section{Induction of C. difficile Enterocolitis and Probiotic Administration}

For the in vivo experiment, B. longum JDM301 and C. difficile ATCC 43255 recovered from $-80^{\circ} \mathrm{C}$ frozen storage were inoculated on agar plates and incubated at $37^{\circ} \mathrm{C}$ under anaerobic condition for $48 \mathrm{~h}$. After $48 \mathrm{~h}$, colonies were inoculated to MRS or BHI, respectively. The cultures were transferred into fresh MRS or BHI at 10\% inoculums for $24 \mathrm{~h}$. Cells were collected by centrifugation at 13,400 rpm for $5 \mathrm{~min}$ and washed twice with sterile PBS. Then bacteria pellets were suspended in sterile PBS. The concentration of $C$. difficile was evaluated according to $\mathrm{OD}_{600}$ and adjusted to $10^{10} \mathrm{CFU} / \mathrm{ml}$ in PBS. And the concentration of $B$. longum was adjusted to $10^{11}$ or $10^{9}$ $\mathrm{CFU} / \mathrm{ml}$. The bacterial counts of $\mathrm{CFU} / \mathrm{ml} / \mathrm{OD}_{600}$ of bacteria incubated at $37^{\circ} \mathrm{C}$ in MRS or BHI for $24 \mathrm{~h}$ in anaerobic conditions were determined by plating serial dilutions of the cultures on MRS-cys/agar $0.05 \%$ (w/v) or BHI-cys/agar $0.05 \%$ $(\mathrm{w} / \mathrm{v})$ and plates were incubated at $37^{\circ} \mathrm{C}$ for $48 \mathrm{~h}$ in anaerobic conditions. C57BL/6 mice (6 7 weeks old) were purchased from Shanghai Laboratory Animal Co., Ltd. (SLAC, China). All animal experiments were approved by the Animal Care and Use Committee of Xuzhou Medical University. The infection model used is a modification of the protocol reported previously (Chen et al., 2008; Kang et al., 2011). C57BL/6 male mice (8 weeks) were given antibiotic-containing drinking water for 3 days. The antibiotic concentrations in drink water were as follows: kanamycin, $1.6 \mathrm{mg} / \mathrm{ml}$; gentamicin, $0.14 \mathrm{mg} / \mathrm{ml}$; colistin, $0.168 \mathrm{mg} / \mathrm{ml}$; metronidazole, $0.86 \mathrm{mg} / \mathrm{ml}$; vancomycin, $0.18 \mathrm{mg} / \mathrm{ml}$. After exposure to the antibiotic cocktail, mice were given untreated autoclaved water for a whole day. Then, mice received a single dose of clindamycin $(10 \mathrm{mg} / \mathrm{kg})$ intraperitoneally. After $24 \mathrm{~h}$, mice were divided into three groups: Control group (no infection and no treatment), CD group (CDI) and $\mathrm{CD}+\mathrm{BF}$ group (CDI and JDM301 treatment). Mice in $\mathrm{CD}$ group and $\mathrm{CD}+\mathrm{BF}$ group were infected with $C$. difficile ATCC $43255\left(1 \times 10^{9} \mathrm{cfu}\right)$ by oral gavage and monitored for signs of disease (day 1). Control mice were similarly administered with $200 \mu \mathrm{l}$ sterile PBS. Mice in CD+BF group received a dose of $1 \times 10^{10} \mathrm{CFU}$ B. longum JDM301 by oral gavage followed administration of $C$. difficile on day 1 . From day 2 to day 6, B. longum JDM301 $\left(1 \times 10^{9} \mathrm{cfu}\right)$ in drinking water was administrated to mice in $\mathrm{CD}+\mathrm{BF}$ group. The clinical endpoint was time until death occurred. During the entire post-infection, clinical scores were recorded for each mouse (Li et al., 2012). A few modifications were made that clinical scores for animals died spontaneously were not included in clinical scoring. On day 7 , surviving animals were sacrificed to collect tissues and cecal content. Samples from animals that died spontaneously were not included in histopathological analysis, cytokine array, quantification of the number, and the toxin of $C$. difficile in gut.

\section{Histopathological Analysis}

For each mouse, cecum was collected and fixed in a $4 \%$ phosphate buffered formaldehyde solution. Paraffin embedded samples were sliced and stained with hematoxylin-eosin for light microscopic examination. Histological damage of cecum segments was evaluated based on a scoring system reported previously (Hirota et al., 2012). A histological scoring system was used to assess pathological tissue inflammation and architectural changes. Architectural change was scored: 0, normal; 1, vacuolation/blebbing; 2, loss of epithelium; and 3, complete loss of crypt architecture. The severity of the inflammatory response 
was scored: 0 , normal; 1 , increased number of inflammatory cells in lamina propria; 2 , increased number of inflammatory cells in submucosa; 3 , dense inflammatory cell mass, but not transmural in nature; and 4, transmural inflammation. The histopathology score for each mouse was a sum of a score for architectural change and an inflammation score.

\section{Cytokine Quantification by Cytometric Bead Array (CBA)}

Proximal cross section of colon $(20 \mathrm{mg}$ ) from the above mice was weighted. Then, $400 \mu \mathrm{l}$ RIPA (Sigma) and protease inhibitor cocktail (Santa Cruz) were added to the samples. Tissues were homogenized on ice and centrifuged at $12,000 \mathrm{~g}$ for $10 \mathrm{~min}$ at $4^{\circ} \mathrm{C}$. Supernatants were collected. CBAs were performed using a CBA mouse Th1/Th2/Th17 cytokine kit (BD Biosciences) with a FACSCanto II flow cytometer to evaluate the concentrations of IL-6, TNF- $\alpha$, IL-10, IL-17, and IFN- $\gamma$ in the supernatants of the tissues. FCAP Array software v 3.0 (BD Biosciences) was used to analyze cytometric data.

\section{Statistical Analysis}

The data are presented as the averages of at least three independent experiments; error bars represent the standard deviations. Differences between multiple groups were compared using ANOVA test with post hoc Bonferroni correction. A Student's $t$-test was used for comparisons between two groups. A $P$-value $<0.05$ was considered significant.

\section{RESULTS}

\section{B. longum JDM301 Inhibits the Growth and Toxin Production of C. difficile in Vitro}

To test whether B. longum JDM301 can inhibit the growth of $C$. difficile, we used a co-culture system in vitro. Our results indicated that at different inoculation ratio (B. longum: C. difficile), the growth of $C$. difficile virulent strain-ATCC 43255 was significantly inhibited (Figure 1A). The inhibitory effect was dose-dependent, as the more B. longum added, the more significant growth inhibition was seen (Figure 1A). Similar findings were obtained in another $C$. difficile strain-ATCC 9689 (Figure 1B). The results together suggested that $B$. longum JDM301 could potentially inhibit $C$. difficile growth when they lived in the same environment.

In many previous reports, the strain ATCC 43255 was used to infect mice to establish mice models of antibioticinduced C. difficile-associated disease, since it was a high-level toxin-producing strain (Chen et al., 2008; Kang et al., 2011; Babakhani et al., 2013). In our study, the strain ATCC 43255 was used to infect C57BL/6 mice in vivo evaluation. Accordingly, the following in vitro experiments related to its main virulence factors-TcdA and TcdB were performed using the strain ATCC 43255. Thus, the levels of TcdA and TcdB, the important toxins to induce the host diseases, in the co-culture system of ATCC 43255 and B. longum JDM301 were determined. Dot-blot assay showed that the reduction of TcdA and TcdB levels depended on the inoculation ratio of $B$. longum JDM301 to $C$. difficile (Figures 2A,B). Similarly, the titers of TcdA (Figure 2C) and TcdB (Figure 2D) from $C$. difficile were reduced significantly in the presence of B. longum JDM301, which was dose-dependent as revealed by ELISA. These results suggested that the levels of clostridial toxins were negatively correlated with the inoculation ratio of B. longum JDM301 to C. difficile and positively correlated with the number of $C$. difficile (Figures 1, 2). Thus, when present together, B. longum JDM301 can reduce clostridial toxin levels by inhibiting the growth of $C$. difficile.

\section{B. longum JDM301 Supernatant Reduces the Toxin Titres of C. difficile}

Further experiments were performed to test whether the CFS of B. longum JDM301 could exert anti-clostridial activity. For this end, the ATCC 43255 strain of C. difficile was inoculated in CFS
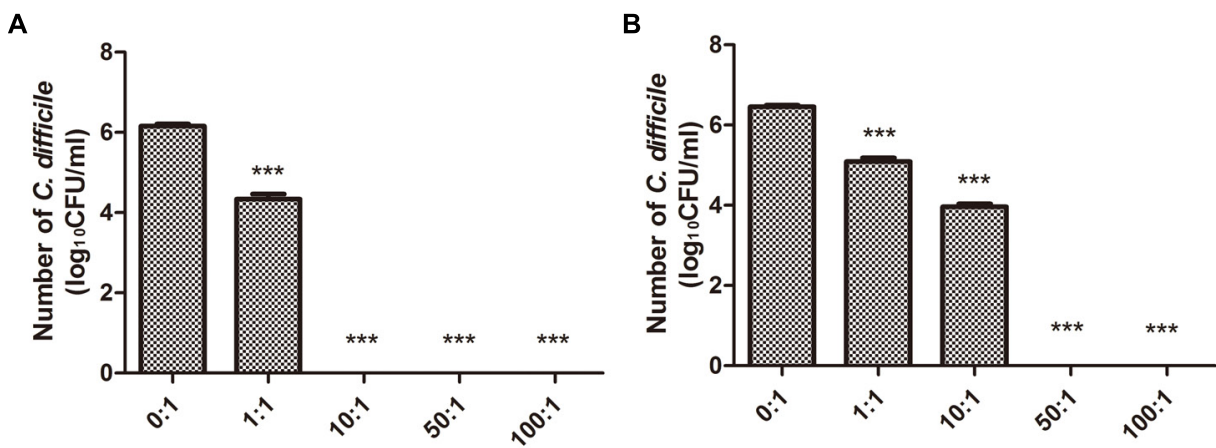

FIGURE 1 | Growth of $C$. difficile co-cultured with B. longum JDM301. (A) The growth of ATCC 43255 co-cultured with B. longum JDM301. C. difficile ATCC 43255 was co-cultured with B. longum JDM301 at different inoculation ratios (B. longum: C. difficile 43255 ) in $\mathrm{BHI}$ for $48 \mathrm{~h}$ at $37^{\circ} \mathrm{C}$ under anaerobic conditions. (B) The growth of ATCC 9689 co-cultured with B. longum JDM301. C. difficile ATCC 9689 was co-cultured with B. longum JDM301 at different inoculation ratios (B. Iongum: C. difficile ATCC 9689) in $\mathrm{BH}$ for $48 \mathrm{~h}$ at $37^{\circ} \mathrm{C}$ under anaerobic conditions. The values presented are the averages of three independent experiments; error bars represent the standard deviations. ${ }^{* * *} P<0.001$ (when $C$. difficile co-cultured with $B$. longum at different inoculation ratios was compared with $C$. difficile cultured alone). 
A Ratio (B1:Ce):

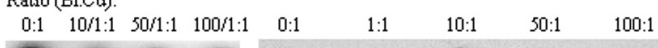

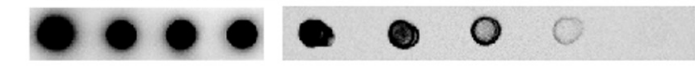

B Ratio (B1:Cc):

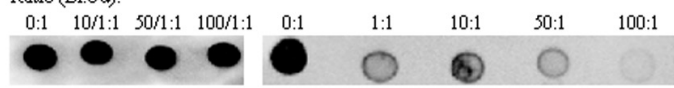

C

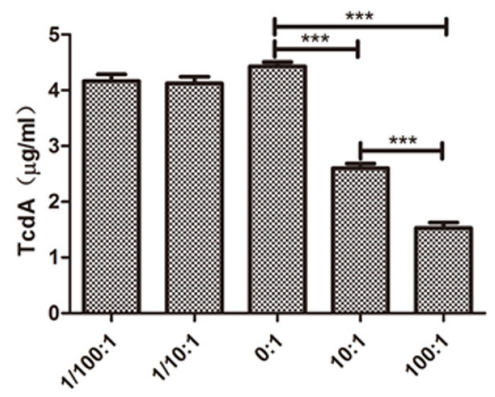

D

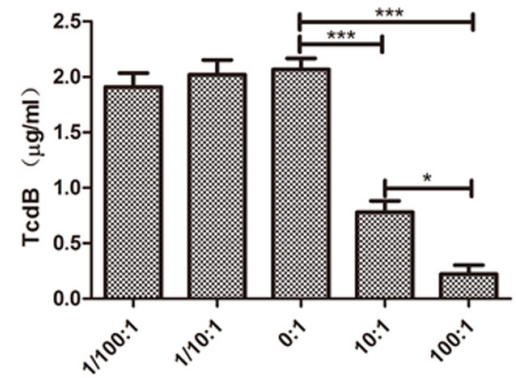

FIGURE 2 | Toxin levels for $C$. difficile strain ATCC 43255. C. difficile ATCC 43255 was co-cultured with $B$. longum JDM301 at different inoculation ratios (B. longum: C. difficile) in BHI supplemented with $0.05 \%(w / v) L-c y s t e i n e-H C l$ for $48 \mathrm{~h}$ at $37^{\circ} \mathrm{C}$ under anaerobic conditions. Samples (1 $\mathrm{ml}$ aliquots) were taken at $48 \mathrm{~h}$ and the supernatants were collected by centrifugation. Then toxins in these supernatants were detected. (A) TcdA was detected by Dot-blot assay. (B) TcdB was detected by Dot-blot assay. The experiments were repeated three times and representative blots were shown. (C) TcdA was detected by ELISA. (D) TcdB was detected by ELISA. The values are presented as the averages of three independent experiments; error bars represent the standard deviations. ${ }^{*} P<0.05$, ${ }^{* * *} P<0.001$ (when $C$. difficile co-cultured with $B$. longum at different inoculation ratios was compared with C. difficile cultured alone)

from B. longum JDM301. The original pH of JDM301 supernatant was about 4.9. Our data showed that when cultured in CFS from B. longum JDM301, the growth of $C$. difficile was significantly reduced compared with those grew in the standard BHI broth (Supplementary Table S2). The inhibitory effect of the CFS from B. longum JDM301 was $\mathrm{pH}$-sensitive, as decreased inhibition was observed when JDM301-supernatant's $\mathrm{pH}$ was adjusted to $\mathrm{pH} 7$ (Supplementary Table S2).

In addition, the CFS of ATCC 43255 was directly incubated with the CFS from B. longum JDM301. Our results showed that the CFS from B. longum JDM301 could directly cause the degradation of $C$. difficile toxin, as both the TcdA and TcdB were undetectable in the $C$. difficile supernatant treated with original CFS ( $\mathrm{pH}=4.9)$ from B. longum JDM301 (Figures 3A-D). Interestingly, when the $\mathrm{pH}$ of CFS from B. longum JDM301 was adjusted to $\mathrm{pH} 7$, the TcdA recovered in significant amount, but at a level still lower than those incubated in the BHI media, whereas the TcdB remained undetectable (Figures 3B,D). The results above implied that some components in the supernatant of JDM301 resulted in the degradation of clostridial toxin and that the degradation of TcdA was promoted by acid $\mathrm{pH}$. When present together, it was suggested that the acid $\mathrm{pH}$ induced by probiotic strains not only could play an important role in the growth inhibition against $C$. difficile resulting in the reduction of toxin titres, but also could directly promote the degradation of clostridial toxin.

\section{Growth Inhibition Against C. difficile by CFS From Different Bifidobacterium Strains and Other Symbiotic Bacteria}

Furthermore, C. difficile 43255 was incubated with CFS from other probiotic strains or candidates (total of 25 bifidobacterial strains and 15 symbiotic bacteria strains from other species), respectively and comparative studies among bifidobacterial strains were performed to reveal the difference among the inhibitory effects against $C$. difficile growth by CFS from these symbiotic bacteria. Results showed that the inhibitory effect of CFS from JDM301 was similar with the other 8 B. longum strains (Figure 7). Of note was that the not neutralized JDM301-CFS showed significant higher inhibitory capacity compared with strain BLY1 (Figure 7A). However, when it was neutralized, the significant different was lost (Figure 7B). No differences were found among six species, including B. longum, B. bifidum, B. animalis, B. pseudocatenulatum, L. casei, and L. plantarum, which may due to the variation within the same species (Supplementary Figures S2A,C). The inhibitory effects by genus Bifidobacterium and Lactobacillus were also compared, since the most common probiotic bacteria were certain strains from the two genera. Similarly, no difference was found between the two genera (Supplementary Figures S2B,D). Since the level of clostridial toxins was positively correlated with the number of $C$. difficile as mentioned above (Figures 1, 2), these results suggested that JDM301 and most of the bifidobacterial strains could (at least partially) reduce toxin titres through growth inhibition against $C$. difficile, which was acid $\mathrm{pH}$ dependent.

\section{B. longum JDM301 Attenuates CDI in Vivo}

To test whether B. longum JDM301 can be used to prevent or treat CDI in vivo, we infected B6 mice with C. difficile 43255 and treated them with B. longum JDM301. There were eight mice died in $\mathrm{CD}$ group, while three mice died in $\mathrm{CD}+\mathrm{BF}$ group before day 7. Survival analysis was performed by Mantel-Cox $(P=0.0215)$. During the 7 -day post-infection period, there was a $43 \%$ survival rate in the mice of CD group, while those treated with $B$. longum JDM301 (CD+BF group) had an increasing survival rate to $75 \%$ (Figure 4C), implying a beneficial effect of B. longum in treating CDI. Furthermore, percent weight loss from day 1 (day of infection) and clinical scores were obtained. B. longum-treated mice showed a relatively less decrease of the body weight and lower clinical scores than the CD group (Figures 4A,B). Microscopically, the B. longum-treated mice 


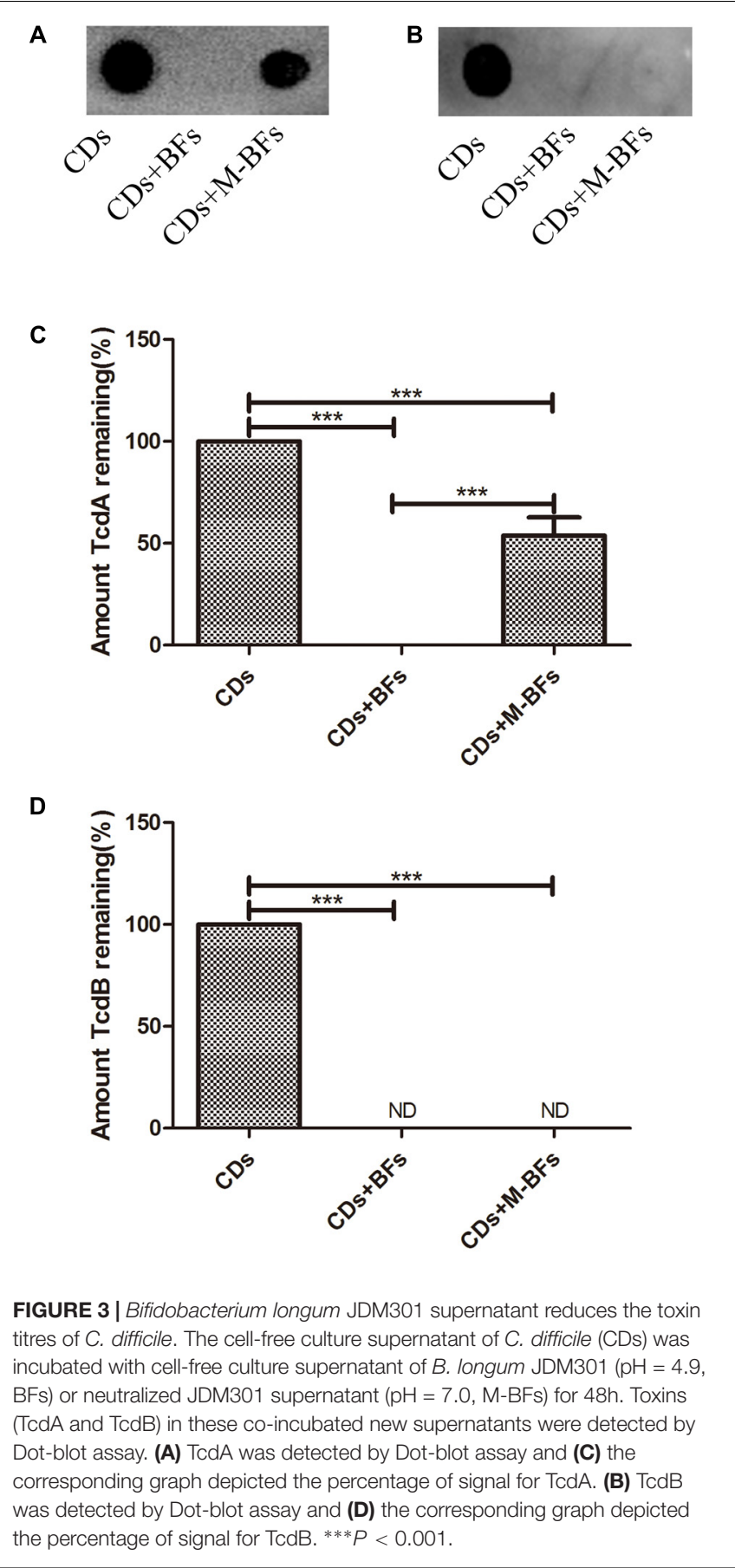

also showed less mucosal damage compared with $\mathrm{CD}$ group (Figures 5A,B).

\section{B. longum JDM301 Reduces Gut Inflammation in the C. difficile-Infected Mouse}

To determine whether $B$. longum JDM301 regulates inflammatory process during CDI, IL-6, IL-17, IFN- $\gamma$, TNF- $\alpha$, and IL-10 levels in the colon tissues were determined accordingly.
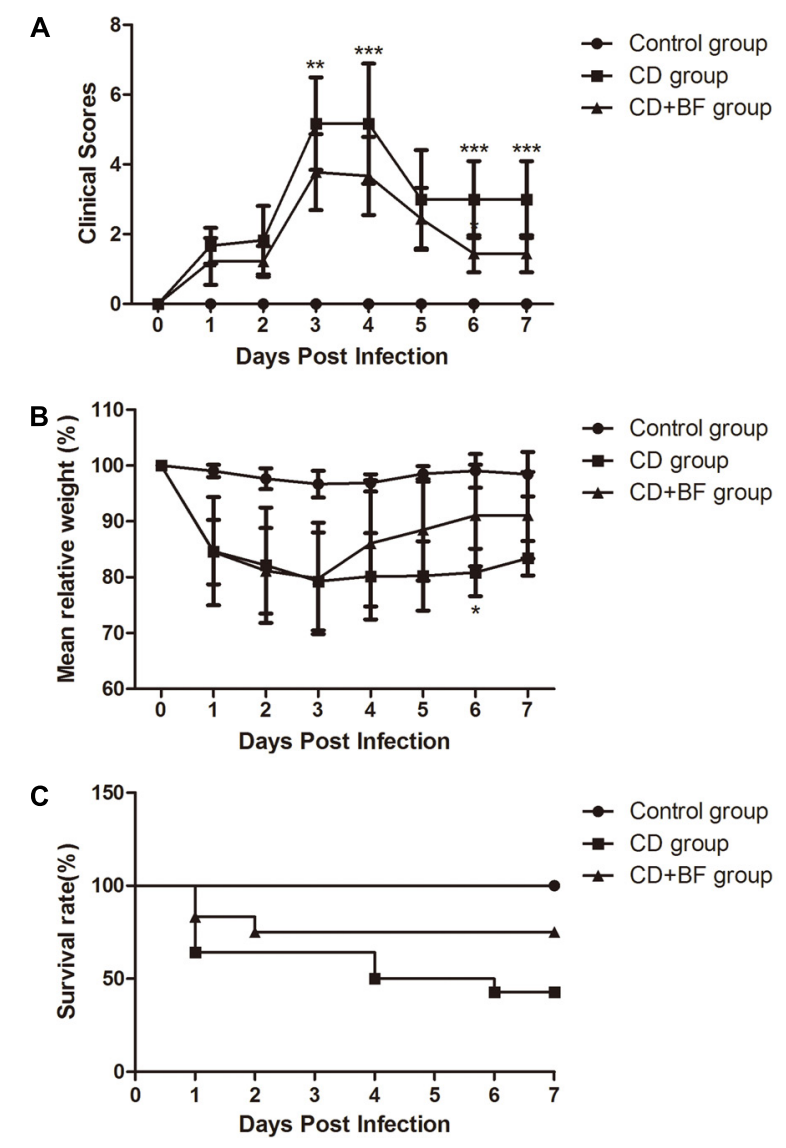

FIGURE 4 | Bifidobacterium longum JDM301 attenuates CDI in vivo. Mice were infected with ATCC 43255 treated with or without B. longum JDM301. (A) Clinical scores. (B) Percent weight loss from day 0 (the day of infection). (C) Survival curve $P=0.0215$ by Mantel-Cox $(n=6-9) .{ }^{*} P<0.05$,

${ }^{* *} P<0.01,{ }^{* * *} P<0.001$.

As shown in Figure 5, significant increases in IL-6 and TNF- $\alpha$ production were found in CDI mice than uninfected mice (Figures 5C,D). While both IL-6 and TNF- $\alpha$ production were significantly reduced in the colon tissues of $B$. longum-treated mice compared with those of untreated mice, which suggested down-regulation of inflammation by B. longum JDM301 treatment (Figures 5C,D). Furthermore, cytokine IL-10 in the colon tissues was significantly increased in the JDM301-treated mice than in the infected but untreated mice (Figure 5E), which indicated that the improved production of IL-10 may be involved in the down-regulation of inflammatory response to CDI by B. longum JDM301. These results suggested that B. longum treatment could temper gut inflammation during CDI.

\section{B. longum JDM301 Treatment Reduces the Number and Toxin of C. difficile in Cecum}

To determine whether JDM301 can inhibit C. difficile growth in vivo, the number of $C$. difficile in the cecal contents was measured by qPCR. As shown in Figure 6, the number of 


\section{A}
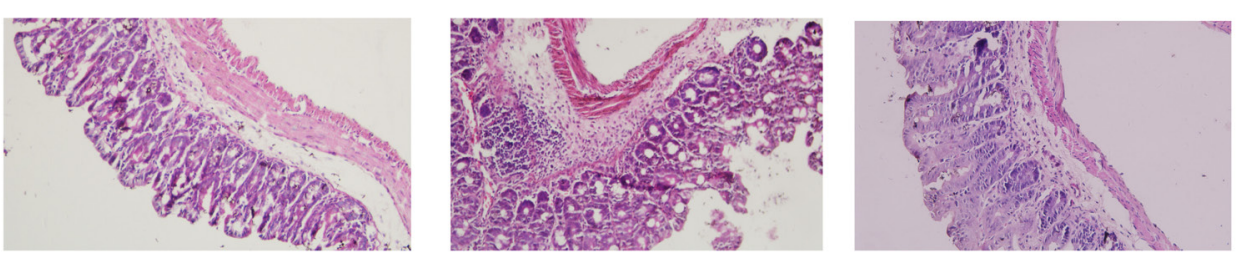

B

C

Infection group
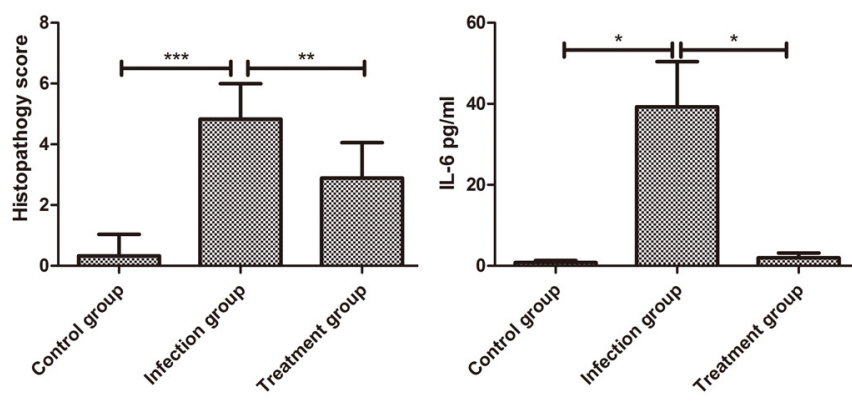

D

Treatment group

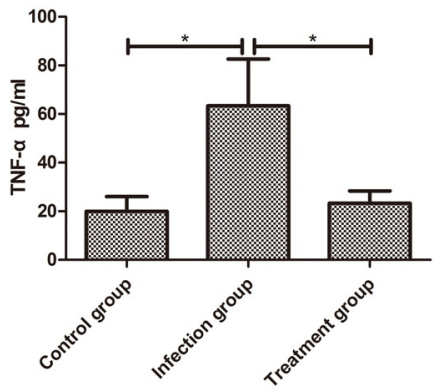

E

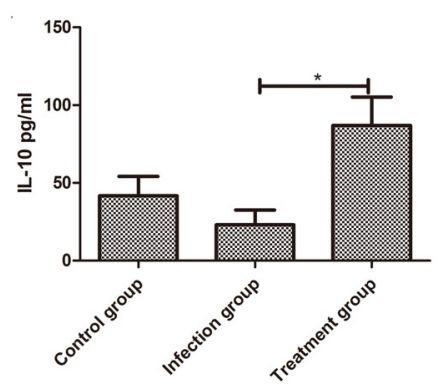

$F$

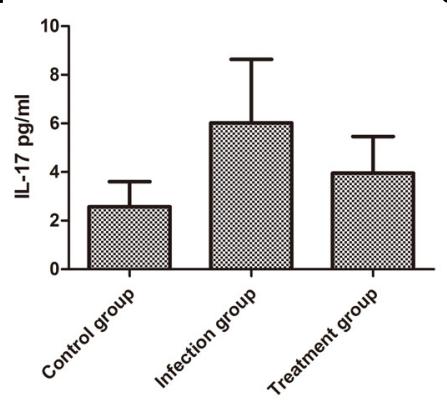

G

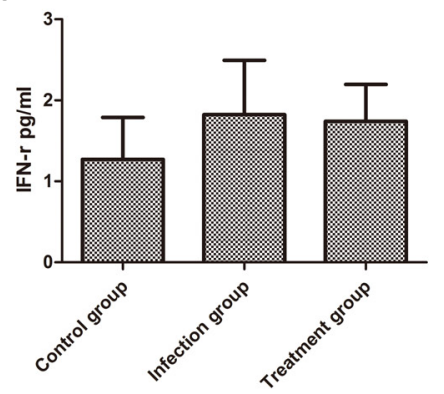

FIGURE 5 | Bifidobacterium longum JDM301 reduces gut inflammation in the C. difficile-infected mouse. On day 7, surviving animals were sacrificed to collect tissues. (A) Representative cecum were stained with hematoxylin and eosin from Control group, CD group, and CD+BF group. (B) Histological assessment of cecum stained with hematoxylin and eosin. (C-G) IL-6, IL-17, IFN- $\gamma$, TNF- $\alpha$, and IL-10 in the proximal cross section of colon were detected by flow cytometry. Data were presented as mean $\pm \mathrm{SD}(n=6-9) .{ }^{*} P<0.05,{ }^{* *} P<0.01,{ }^{* * *} P<0.001$.

C. difficile dramatically decreased in the cecal content of B. longum-treated mice than that of untreated mice (Figure 6A). TcdA and TcdB levels were also significantly reduced in the $B$. longum-treated mice (Figures $\mathbf{6 B}, \mathbf{C}$ ). These data were consistent with our in vitro culture data described above and strongly supported of a potential application of B. longum JDM301 in preventing or treating CDI.

\section{DISCUSSION}

Clostridium difficile is considered an important cause of antibiotic associated diarrhea in human. Two large protein toxins, TcdA and $\mathrm{TcdB}$ are the main virulence factors of $C$. difficile (Basseres et al., 2016). The standard antibiotic therapies (metronidazole and vancomycin) used in the treatment for CDI, are limited by the perturbation of intestinal flora due to their broad spectrum. The perturbation of intestinal flora can result in high recurrence rate of CDI. For probiotics, they can restore the complex balance of the indigenous microbiota in gut. Moreover, it is less possible for probiotics to increase the antibiotic resistance of $C$. difficile.
Thus, probiotics may be used as alternative strategy to preventing or treating CDI.

Some strains of $B$. longum are able to inhibit the growth and adhesion of pathogen to human epithelial cells, to modify gut flora and to modulate host immune response (Benno and Mitsuoka, 1992; Xu et al., 2009; Yang et al., 2015). It is believed that the indigenous gut microbiota involves in the resistance against colonization by bacterial pathogens, including C. difficile. The baseline differences in microbiota of mice used to establish CDI model might cause differences in results (Britton and Young, 2011). Pre-exposure to antibiotics is required for productive infection of animals with $C$. difficile, which was performed in our study as previously reported (Chen et al., 2008). Antibiotic administration alters the gut microbiota, which permits germination of $C$. difficile spores and expansion of the pathogen (Britton and Young, 2011). Previous reports showed that supplementation of probiotics contributed to restore the gut microbial diversity (Vemuri et al., 2017). Grazul et al. (2016) showed the potential mechanism that probiotics remodeled the microbiome of host recovering from antibiotic therapy, which may be involved in the alleviation of damage in CDI mice by 


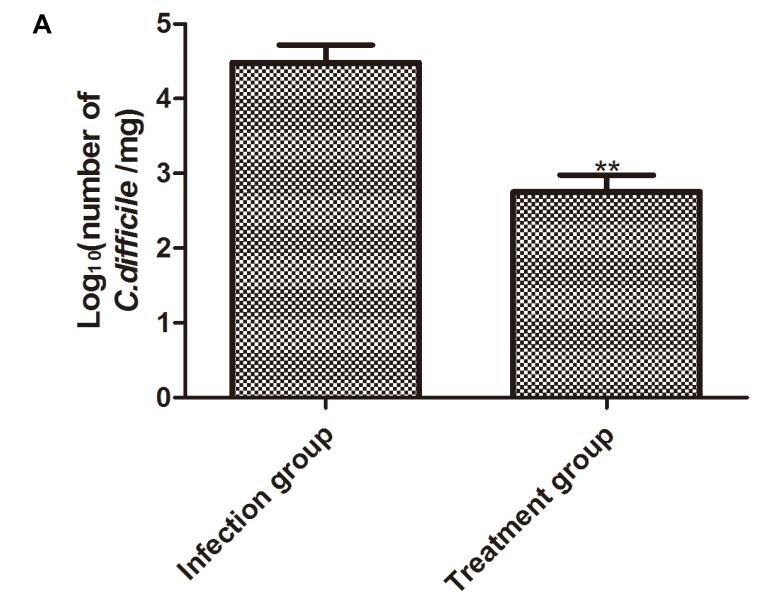

B

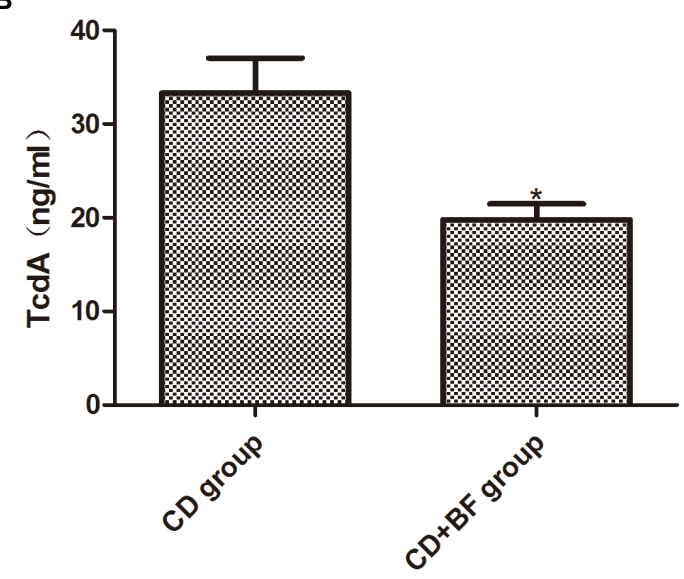

C

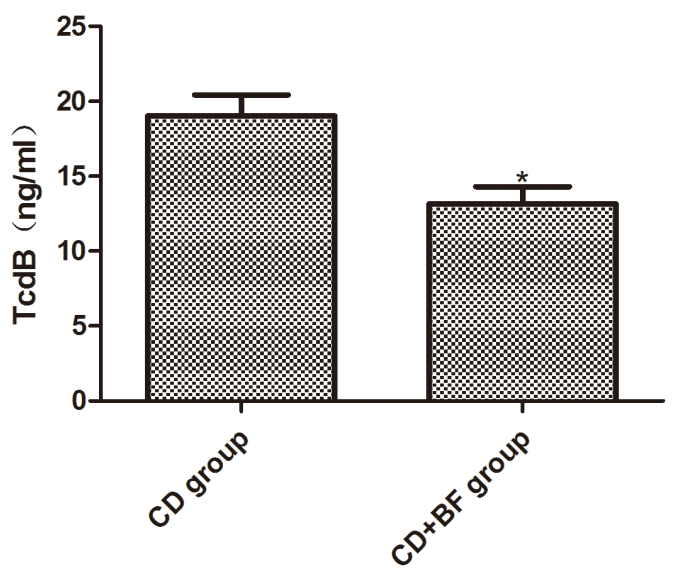

FIGURE 6 | Bifidobacterium longum JDM301 treatment reduces the number and toxin of $C$. difficile in cecum. Cecal contents were obtained on the day 7 post-infection. (A) The number of $C$. difficile in cecal contents was measured by qPCR. (B,C) TcdA and TcdB levels in cecal contents were determined by commercially available ELISA kits. Data were presented as mean \pm SD $(n=6-9),{ }^{*} P<0.05,{ }^{* *} P<0.01$.

JDM301 treatment in this study. In other cases, FMT was used to overcome CDI through therapeutic restoration of a diverse microbiota (Hudson et al., 2017).

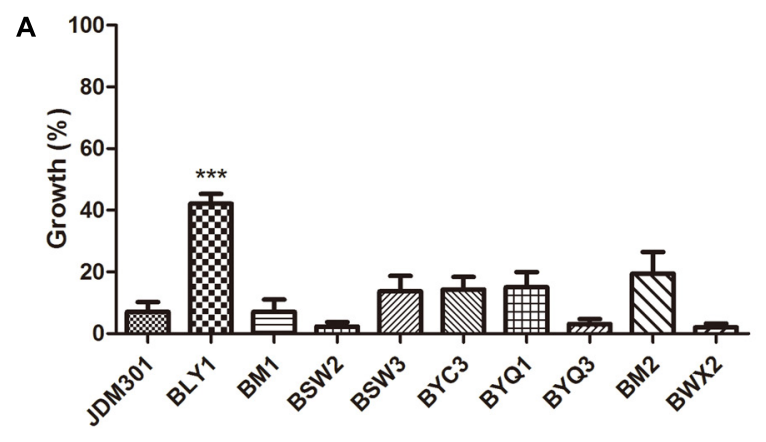

B

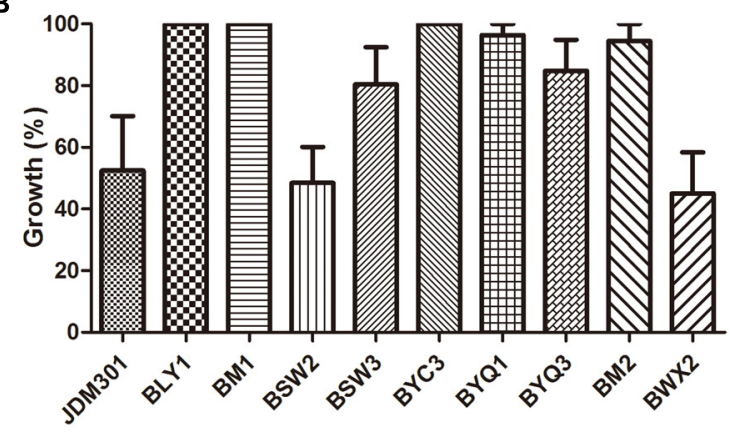

FIGURE 7 | Growth inhibition against $C$. difficile by CFS from different B. longum strains. C. difficile was cultured anaerobically in CFS from 10 B. longum strains at $37^{\circ} \mathrm{C}$ for $24 \mathrm{~h}$. The percentage of growth was calculated by comparing the final $\mathrm{OD}_{600}$ obtained with $C$. difficile cultured in CFS of different $B$. longum strains with those of the corresponding control samples [C. difficile cultured in BHI-cys $0.05 \%(\mathrm{w} / \mathrm{V})]$. (A) The effect of original CFS from $B$. longum on growth of $C$. difficile. (B) The effect of $\mathrm{pH}$-modified CFS from the corresponding strains on growth of $C$. difficile. ${ }^{* * *} P<0.001$ (when the effect of CFS from BLY1 was compared with the effect of CFS from JDM301).

So far, the role of probiotics in prevention of CDI is still controversial. The efficacy to prevent $C$. difficile and mechanisms underlying the antibacterial activity are strain dependent (Chenoll et al., 2011; Kalil and Schooneveld, 2014). Utilization of different probiotics on varied patient may be responsible for the conflicting data on probiotics for the prevention of CDI reported previously (Mills et al., 2018). Thus, it is needed to select the optimal probiotic strain or combination of strains for prevention of CDI. Yun et al. (2017) co-cultured one B. longum strain (ATCC 15707) with a C. difficile 027 strain in vitro and investigated the effect of ATCC 15707 on the mortality and intestinal damage through survival rates and histopathological analysis. They reported that ATCC 15707 treatment alleviated C. difficile induced damage revealed by histopathological analysis and improved survival rate (Yun et al., 2017). Differently, in our study, the anti-clostridial effects of B. longum JDM301 (JDM301), a widely used commercial probiotic strain in China, were evaluated comprehensively in vitro and in vivo, which was combined with comparative studies involving 40 strains of probiotic strains or candidates to reveal the anti-clostridial effects of Bifidobacterium. B. longum JDM301 was chosen for its wide use in industry as probiotics in China (Wei et al., 2017). Because TcdA and TcdB from C. difficile are the main 
virulence factors of this pathogen, the anti-toxin (TcdA and $\mathrm{TcdB}$ ) capability of $B$. longum was investigated both in vitro and in vivo. In vitro evaluation revealed that B. longum JDM301 reduced TcdA and TcdB titres by both inhibiting the growth of $C$. difficile and promoting the degradation of clostridial toxin. Additionally, the results of in vitro experiments were confirmed by determining TcdA and TcdB titres and detecting the number of C. difficile in cecal contents of CDI mice. Notably, the result showed that the degradation of $C$. difficile TcdA by CFS from JDM301, was promoted by acid $\mathrm{pH}$. To our knowledge, there has been no evidence on the degradation of C. difficile toxin promoted by acid $\mathrm{pH}$, when the toxin was incubated in CFS from probiotics in previous reports. The in vitro evaluation suggested that the reduced level of clostridial toxin could result from the growth inhibition by probiotics and the degradation of clostridial toxin by CFS from probiotics. Then, an in vivo test was carried out using a mouse model of CDI. Comprehensive evaluation of inflammatory response induced by $C$. difficile was performed using CDI mice in our study. CDI mouse exhibited a significant increase of IL-6 production, a marker of acute inflammation as previously reported (Kim et al., 2007; Kang et al., 2011), but IL-6 production was sharply reduced by B. longum JDM301 treatment. Similarly, the production of another inflammatory cytokine, TNF- $\alpha$ increased significantly in CDI mice, but B. longum JDM301 treatment reduced its production. Inflammatory cytokines, IL-6 and TNF- $\alpha$ were both decreased by JDM301 treatment, while immunosuppressive cytokine, IL-10 increased in gut of JDM301treated mice compared with untreated mice. These results suggested that the inflammatory response to CDI was tempered by B. longum JDM301. In previous reports, some bifidobacterial strains were able to ameliorate inflammation by inducing IL10 production (Jeon et al., 2012). These results indicated a role of improved IL-10 production in the down-regulation of inflammatory response to CDI by B. longum JDM301. Relative body weight changes and clinical scores were analyzed. These results combined with histopathological analysis and cytokine quantification of gut tissues revealed that B. longum JDM301 treatment attenuated the damage induced by CDI. Together with the significant reduction of clostridial number and toxin in CDI mice treated with bifidobacteria, these results suggested that strain B. longum JDM301 exerted antagonistic activity against C. difficile and relieved the damage of enteric tissues caused by this pathogen in vivo.

Comparative studies among $10 \mathrm{~B}$. longum strains, or 6 species belonging to Bifidobacterium and Lactobacillus or the two genera were performed to reveal the difference among the growth inhibition against $C$. difficile of CFS from 40 strains of symbiotic bacteria, including 25 bifidobacterial strains, respectively. So far, there are only a few comparative studies among different probiotic strains or species. Trejo et al. (2010) co-cultivated C. difficile 9689 or a clinical isolate with 25 bifidobacteria or lactobacilli and they found that the capability to antagonize the toxic effect upon Vero line was strain dependent. ValdesVarela et al. (2016) incubated 20 probiotic strains with a toxigenic supernatant from C. difficile LMG21717 and analyzed the effect of the supernatant upon the biological model HT29.
They found a species-efficacy association with the anti-toxin capability to protect HT29 cells from the cytotoxicity caused by toxigenic C. difficile supernatants (Valdes-Varela et al., 2016). The experimental used in our study was based on the incubation of C. difficile 43255 with CFS from 40 probiotic strains or candidates, respectively (Supplementary Table S2). Results showed that the inhibitory effect of CFS from JDM301 was similar with the other $8 \mathrm{~B}$. longum strains and significant higher than strain BLY1. In our study, JDM301 also demonstrated antagonistic activity against $C$. difficile in co-culture system. In addition, no differences were found among these species or between genus Bifidobacterium and Lactobacillus. These results showed that CFS from JDM301 and most of the bifidobacterial strains were able to inhibit the growth of $C$. difficile and that the inhibition effect induced by CFS from probiotic strains or candidates depended on acid pH (Supplementary Figure S1). Since the level of clostridial toxins was positively correlated with the number of $C$. difficile (Figures 1, 2), these results suggested that JDM301 and most of the bifidobacterial strains could (at least partially) exert protective effects by reducing toxin titres through growth inhibition against $C$. difficile. It was suggested that the acid $\mathrm{pH}$ induced by probiotic strains not only was involved in the growth inhibition against $C$. difficile leading to the reduction of toxin titres, but also directly promoted the degradation of clostridial toxin. Additionally, probiotics have been redefined as "Live microorganisms which when administered in adequate amounts confer a health benefit on the host" (FAO/WHO, 2001). In our case, the antagonistic activity of JDM301 against $C$. difficile was dose-depended and the adequate amounts were also needed for JDM301 to exert anti-clostridial activity, when JDM301 and $C$. difficile were co-cultured. Overall, our results provided a comprehensive insight into the anti-clostridial activity of Bifidobacterium strains, including growth inhibition, degradation of clostridial toxin by CFS from probiotics, the down-modulatory of inflammation, the reduction of clostridial number and the decreased toxin titres in cecal contents of CDI mice.

In order to determine whether there were some components in B. longum JDM301 CFS able to directly cause the degradation of TcdA and TcdB, the toxigenic CFS of ATCC 43255 was incubated directly with the CFS from B. longum JDM301. Our results showed that the levels of $C$. difficile toxins decreased sharply when compared with these in C. difficile CFS co-incubated with BHI broth (Control group). Interestingly, the TcdA concentration increased in $C$. difficile supernatant treated with neutralized JDM301 CFS, but still lower than Control group, while TcdB was undetectable in both the toxigenic CFS from $C$. difficile incubated with the original CFS and the toxigenic CFS incubated with the neutralized CFS from B. longum JDM301. These results suggested that the acid $\mathrm{pH}$ could promote the degradation of C. difficile TcdA by CFS from JDM301. However, the degradation of TcdB seems to be less $\mathrm{pH}$-sensitive. These results implied that some molecules secreted by JDM301 were able to directly reduce toxin titres by degrading clostridial toxins and that the low $\mathrm{pH}$ may play different roles in the degradation of $\mathrm{Tcd} A$ and $\mathrm{TcdB}$ by CFS from JDM301. Genes encoding exo-peptidases may be involved in the degradation of $C$. difficile toxins by CFS from JDM301 (Wei et al., 2010). So far, only a few peptidases from 
bifidobacteria have been described and were strain specific (Meli et al., 2013). In previous report, L. lactis subsp. lactis CIDCA 8221 protected vero cells from $C$. difficile toxins by secreting some heat-sensitive products (Bolla et al., 2013). Saccharomyces boulardii could release an extracellular serine-protease able to degrade the TcdA (Pothoulakis et al., 1993). Many studies have focused on the metabolites involving the antagonistic activities of probiotics against human pathogens, including $C$. difficile. The strictly fermentative sugar metabolism of bifidobacteria produces organic acids, such as lactate, acetate, formate, and so on, which generates the low pH (Falony et al., 2006). Schoster et al. (2013) showed that probiotic strains inhibited clostridial growth in a $\mathrm{pH}$-dependent manner. Notably, our results suggested that the low $\mathrm{pH}$ could promote the degradation of $C$. difficile TcdA. Valdes-Varela et al. (2016) showed that the reduction of TcdA concentration seemed to be correlated with higher protective effect than $\mathrm{TcdB}$, which implied the importance of the totally new role of acid $\mathrm{pH}$ in protective effect of bifidobacteria against toxigenic $C$. difficile. Thus, when present together, our results suggested that the acid $\mathrm{pH}$ induced by probiotic strains not only could play an important role in the growth inhibition against C. difficile leading to the reduction of toxin titres, but also could directly promote the degradation of clostridial toxin. Further study was needed to reveal the mechanism of the degradation of clostridial toxin promoted by low $\mathrm{pH}$.

In the current study, the in vitro anti-clostridial activity of B. longum JDM301 and its efficacy to prevent CDI in a mouse model were evaluated. B. longum JDM301 can not only inhibit the growth of $C$. difficile but also attenuate its bioactivity by secreting some molecules that can degrade its toxins. Especially, the new role of acid $\mathrm{pH}$ in anti-clostridial activity of probiotics by promoting degradation of clostridial toxin was revealed. In vivo studies using a mouse model have showed that B. longum JDM301 decreases the number and toxin level of $C$. difficile in gut and partially relieves the damage to gastric tissues caused by $C$. difficile. Our results demonstrated that the commercial strain, JDM301 could be considered a probiotic able to exert anti-toxin capability in vitro and in vivo and that most of bifidobacterial strains could (at least partially) exert protective effects by reducing toxin titres through growth inhibition against toxigenic $C$. difficile.

\section{AUTHOR CONTRIBUTIONS}

YxW, XG, KZ, and RT conceived and designed the experiments. YxW, FY, QW, JG, WL, SS, and CL performed the experiments. YxW, FY, BZ, YK, and YgW analyzed the data. YxW was in charge

\section{REFERENCES}

Anand, R., Song, Y., Garg, S., Girotra, M., Sinha, A., Sivaraman, A., et al. (2017). Effect of aging on the composition of fecal microbiota in donors for FMT and its impact on clinical outcomes. Dig. Dis. Sci. 62, 1002-1008. doi: 10.1007/s10620017-4449-6

Babakhani, F., Bouillaut, L., Sears, P., Sims, C., Gomez, A., and Sonenshein, A. L. (2013). Fidaxomicin inhibits toxin production in Clostridium difficile. J. Antimicrob. Chemother. 68, 515-522. doi: 10.1093/jac/dks450 of writing the drafted manuscript. All authors read and approved the final version.

\section{FUNDING}

This work was funded by the National Natural Science Foundation of China (31300029), the Natural Science Foundation of Jiangsu Province, China (BK20130213), the Scientific Research Foundation for the Talents of Xuzhou Medical University (D2012014), the Program for Youth Science and Technology Innovative Research Team of Xuzhou Medical University, a Project Funded by the Priority Academic Program Development of Jiangsu Higher Education Institutions (PAPD) and was sponsored by the Qing-Lan Project of Jiangsu Province, China.

\section{SUPPLEMENTARY MATERIAL}

The Supplementary Material for this article can be found online at: https://www.frontiersin.org/articles/10.3389/fmicb. 2018.00888/full\#supplementary-material

FIGURE S1 | The correlation between the $\mathrm{pH}$ of cell-free culture supernatants (CFS) of different symbiotic bacteria and the growth of $C$. difficile in CFS.

Pearson's correlation was used to analyze the associations between the $\mathrm{pH}$ of the CFS of different symbiotic bacteria. Bifidobacterial strains and other bacteria were cultured at $37^{\circ} \mathrm{C}$ for $24 \mathrm{~h}$ anaerobically. After $24 \mathrm{~h}$, the supernatants were obtained by centrifugation and the $\mathrm{pH}$ was determined. Then, the supernatants were filter-sterilized using $0.22 \mu \mathrm{m}$ filters. C. difficile was cultured in CFS and the growth was measured spectrophotometrically at $24 \mathrm{~h}$ after anaerobic incubation at $37^{\circ} \mathrm{C}$. The percentage of growth was calculated by comparing the final $\mathrm{OD}_{600}$ obtained with $C$. difficile growing in CFS of different symbiotic bacteria, with those of the corresponding control samples [C. difficile cultured in BHI-cys $0.05 \%(\mathrm{~W} / \mathrm{v})$ for $24 \mathrm{~h}$.

FIGURE S2 | Growth inhibition aganist C. difficile by CFs from different species and genera belong to Bifidobacterium and Lactobacillus. C. difficile was cultured anaerobically in CFs from probiotic strains or candidates at $37^{\circ} \mathrm{C}$ for $24 \mathrm{~h}$. The percentage of growth was calculated by comparing the final $\mathrm{OD}_{600}$ obtained with C. difficile cultured in CFs of different symbiotic bacteria with those of the corresponding control samples [C. difficile cultured in BHI-cys 0.05\% (W/v)]. (A) The effect of original CFs from bifidobacterial or lactobacillal species on growth of $C$. difficile. (B) The effect of original CFs from genus Bifidobacterium and Lactobacillus on growth of $C$. difficile. (C) The effect of $\mathrm{pH}$-modified CFs from bifidobacterial or lactobacillal species on growth of $C$. difficile. (D) The effect of $\mathrm{pH}$-modified CFs from genus Bifidobacterium and Lactobacillus on growth of C. difficile.

TABLE S1 | Strains used in this study.

TABLE S2 | Inhibition activity of cell-free supernatant of Bifidobacterium and other symbiotic bacteria against C. difficile.

Badger, V. O., Ledeboer, N. A., Graham, M. B., and Edmiston, C. E. Jr. (2012). Clostridium difficile: epidemiology, pathogenesis, management, and prevention of a recalcitrant healthcare-associated pathogen. JPEN J. Parenter. Enteral. Nutr. 36, 645-662. doi: 10.1177/0148607112446703

Bartlett, J. G. (2002). Clinical practice. Antibiotic-associated diarrhea. N. Engl. J. Med. 346, 334-339. doi: 10.1056/NEJMcp011603

Basseres, E., Endres, B. T., Khaleduzzaman, M., Miraftabi, F., Alam, M. J., Vickers, R. J., et al. (2016). Impact on toxin production and cell morphology in Clostridium difficile by ridinilazole (SMT19969), a novel treatment for C. 
difficile infection. J. Antimicrob. Chemother. 71, 1245-1251. doi: 10.1093/jac/ dkv498

Benno, Y., and Mitsuoka, T. (1992). Impact of Bifidobacterium longum on human fecal microflora. Microbiol. Immunol. 36, 683-694. doi: 10.1111/j.1348-0421. 1992.tb02071.x

Bolla, P. A., Carasi, P., Serradell Mde, L., and De Antoni, G. L. (2013). Kefir-isolated Lactococcus lactis subsp. lactis inhibits the cytotoxic effect of Clostridium difficile in vitro. J. Dairy Res. 80, 96-102. doi: 10.1017/S0022029912000623

Boonma, P., Spinler, J. K., Venable, S. F., Versalovic, J., and Tumwasorn, S. (2014). Lactobacillus rhamnosus L34 and Lactobacillus casei L39 suppress Clostridium difficile-induced IL-8 production by colonic epithelial cells. BMC Microbiol. 14:177. doi: 10.1186/1471-2180-14-177

Britton, R. A., and Young, V. B. (2011). Role of the intestinal microbiota in resistance to colonization by Clostridium difficile. Gastroenterology 146, 1547-1553. doi: 10.1053/j.gastro.2014.01.059

Carter, G. P., Chakravorty, A., Pham Nguyen, T. A., Mileto, S., Schreiber, F., Li, L., et al. (2015). Defining the Roles of TcdA and TcdB in localized gastrointestinal disease, systemic organ damage, and the host response during Clostridium difficile infections. mBio 6:e00551-15. doi: 10.1128/mBio. 00551-15

Carter, G. P., Douce, G. R., Govind, R., Howarth, P. M., Mackin, K. E., Spencer, J., et al. (2011). The anti-sigma factor TcdC modulates hypervirulence in an epidemic BI/NAP1/027 clinical isolate of Clostridium difficile. PLoS Pathog. 7:e1002317. doi: 10.1371/journal.ppat.1002317

Chang, J. Y., Antonopoulos, D. A., Kalra, A., Tonelli, A., Khalife, W. T., Schmidt, T. M., et al. (2008). Decreased diversity of the fecal Microbiome in recurrent Clostridium difficile-associated diarrhea. J. Infect. Dis. 197, 435-438. doi: $10.1086 / 525047$

Chen, X., Katchar, K., Goldsmith, J. D., Nanthakumar, N., Cheknis, A., Gerding, D. N., et al. (2008). A mouse model of Clostridium difficileassociated disease. Gastroenterology 135, 1984-1992. doi: 10.1053/j.gastro.2008. 09.002

Chenoll, E., Casinos, B., Bataller, E., Astals, P., Echevarria, J., Iglesias, J. R., et al. (2011). Novel probiotic Bifidobacterium bifidum CECT 7366 strain active against the pathogenic bacterium Helicobacter pylori. Appl. Environ. Microbiol. 77, 1335-1343. doi: 10.1128/AEM.01820-10

Darkoh, C., DuPont, H. L., Norris, S. J., and Kaplan, H. B. (2015). Toxin synthesis by Clostridium difficile is regulated through quorum signaling. mBio 6:e0256914. doi: $10.1128 / \mathrm{mBio} .02569-14$

Evans, C. T., and Johnson, S. (2015). Prevention of Clostridium difficile infection with probiotics. Clin. Infect. Dis. 60(Suppl. 2), S122-S128. doi: 10.1093/cid/ civ138

Falony, G., Vlachou, A., Verbrugghe, K., and De Vuyst, L. (2006). Cross-feeding between Bifidobacterium longum BB536 and acetate-converting, butyrateproducing colon bacteria during growth on oligofructose. Appl. Environ. Microbiol. 72, 7835-7841. doi: 10.1128/AEM.01296-06

FAO/WHO (2001). Report on Joint FAO/WHO Expert Consultation on Evaluation of Health and Nutritional Properties of Probiotics in Food Including Powder Milk with Live Lactic acid Bacteria. Available at: https://www.mendeley. com/research-papers/health-nutritional-properties-probiotics-food-includingpowder-milk-live-lactic-acid-bacteria/

Geeraerts, S., Ducatelle, R., Haesebrouck, F., and Van Immerseel, F. (2015). Bacillus amyloliquefaciens as prophylactic treatment for Clostridium difficileassociated disease in a mouse model. J. Gastroenterol. Hepatol. 30, 1275-1280. doi: 10.1111/jgh.12957

Giel, J. L., Sorg, J. A., Sonenshein, A. L., and Zhu, J. (2010). Metabolism of bile salts in mice influences spore germination in Clostridium difficile. PLoS One 5:e8740. doi: 10.1371/journal.pone.0008740

Goldenberg, J. Z., Yap, C., Lytvyn, L., Lo, C. K., Beardsley, J., Mertz, D., et al. (2017). Probiotics for the prevention of Clostridium difficile-associated diarrhea in adults and children. Cochrane Database Syst. Rev. 12:CD006095. doi: 10.1002/ 14651858.CD006095.pub4

Grazul, H., Kanda, L. L., and Gondek, D. (2016). Impact of probiotic supplements on microbiome diversity following antibiotic treatment of mice. Gut Microbes 7, 101-114. doi: 10.1080/19490976.2016.1138197

Hirota, S. A., Iablokov, V., Tulk, S. E., Schenck, L. P., Becker, H., Nguyen, J., et al. (2012). Intrarectal instillation of Clostridium difficile toxin A triggers colonic inflammation and tissue damage: development of a novel and efficient mouse model of Clostridium difficile toxin exposure. Infect. Immun. 80, 4474-4484. doi: 10.1128/IAI.00933-12

Hudson, L. E., Anderson, S. E., Corbett, A. H., and Lamb, T. J. (2017). Gleaning insights from fecal microbiota transplantation and probiotic studies for the rational design of combination microbial therapies. Clin. Microbiol. Rev. 30, 191-231. doi: 10.1128/CMR.00049-16

Jeon, S. G., Kayama, H., Ueda, Y., Takahashi, T., Asahara, T., Tsuji, H., et al. (2012). Probiotic Bifidobacterium breve induces IL-10-producing Tr1 cells in the colon. PLoS Pathog. 8:e1002714. doi: 10.1371/journal.ppat.1002714

Juszczuk, K., Grudlewska, K., Mikucka, A., and Gospodarek, E. (2017). Fecal microbiota transplantation - methods of treatment of recurrent Clostridium difficile infections and other diseases. Postepy Hig. Med. Dosw. 71, 220-226. doi: 10.5604/01.3001.0010.3807

Kalil, A. C., and Schooneveld, T. C. (2014). Probiotics and antibiotic-associated diarrhoea. Lancet 383, 29-30. doi: 10.1016/S0140-6736(13)62734-8

Kang, J. K., Hwang, J. S., Nam, H. J., Ahn, K. J., Seok, H., Kim, S. K., et al. (2011). The insect peptide coprisin prevents Clostridium difficile-mediated acute inflammation and mucosal damage through selective antimicrobial activity. Antimicrob. Agents Chemother. 55, 4850-4857. doi: 10.1128/AAC.00177-11

Kim, H., Rhee, S. H., Pothoulakis, C., and Lamont, J. T. (2007). Inflammation and apoptosis in Clostridium difficile enteritis is mediated by PGE2 up-regulation of Fas ligand. Gastroenterology 133, 875-886. doi: 10.1053/j.gastro.2007. 06.063

Kociolek, L. K., and Gerding, D. N. (2016). Breakthroughs in the treatment and prevention of Clostridium difficile infection. Nat. Rev. Gastroenterol. Hepatol. 13, 150-160. doi: 10.1038/nrgastro.2015.220

Kumar, M., Dhaka, P., Vijay, D., Vergis, J., Mohan, V., Kumar, A., et al. (2016). Antimicrobial effects of Lactobacillus plantarum and Lactobacillus acidophilus against multidrug-resistant enteroaggregative Escherichia coli. Int. J. Antimicrob. Agents 48, 265-270. doi: 10.1016/j.ijantimicag.2016.05.014

Lee, I. A., Bae, E. A., Lee, J. H., Lee, H., Ahn, Y. T., Huh, C. S., et al. (2009). Bifidobacterium longum HY8004 attenuates TNBS-induced colitis by inhibiting lipid peroxidation in mice. Inflamm. Res. 59, 359-368. doi: 10.1007/s00011009-0108-5

Leslie, J. L., Huang, S., Opp, J. S., Nagy, M. S., Kobayashi, M., Young, V. B., et al. (2015). Persistence and toxin production by Clostridium difficile within human intestinal organoids result in disruption of epithelial paracellular barrier function. Infect. Immun. 83, 138-145. doi: 10.1128/IAI.02561-14

Li, Y., Figler, R. A., Kolling, G., Bracken, T. C., Rieger, J., Stevenson, R. W., et al. (2012). Adenosine A2A receptor activation reduces recurrence and mortality from Clostridium difficile infection in mice following vancomycin treatment. BMC Infect. Dis. 12:342. doi: 10.1186/1471-2334-12-342

Louh, I. K., Greendyke, W. G., Hermann, E. A., Davidson, K. W., Falzon, L., Vawdrey, D. K., et al. (2017). Clostridium Difficile infection in acute care hospitals: systematic review and best practices for prevention. Infect. Control Hosp. Epidemiol. 38, 476-482. doi: 10.1017/ice.2016.324

Mani, N., and Dupuy, B. (2001). Regulation of toxin synthesis in Clostridium difficile by an alternative RNA polymerase sigma factor. Proc. Natl. Acad. Sci. U.S.A. 98, 5844-5849. doi: 10.1073/pnas.101126598

McDonald, L. C., Killgore, G. E., Thompson, A., Owens, R. C. Jr., Kazakova, S. V., Kazakova, S. V., et al. (2005). An epidemic, toxin gene-variant strain of Clostridium difficile. N. Engl. J. Med. 353, 2433-2441. doi: 10.1056/ NEJMoa051590

Meli, F., Lazzi, C., Neviani, E., and Gatti, M. (2013). Effect of protein hydrolizates on growth kinetics and aminopeptidase activities of some Bifidobacterium species. Anaerobe 22, 130-133. doi: 10.1016/j.anaerobe.2013.05.003

Mills, J. P., Rao, K., and Young, V. B. (2018). Probiotics for prevention of Clostridium difficile infection. Curr. Opin. Gastroenterol. 34, 3-10. doi: 10.1097/ MOG.0000000000000410

Munoz, J. A., Chenoll, E., Casinos, B., Bataller, E., Ramon, D., Genoves, S., et al. (2011). Novel probiotic Bifidobacterium longum subsp. infantis CECT 7210 strain active against rotavirus infections. Appl. Environ. Microbiol. 77, 8775-8783. doi: 10.1128/AEM.05548-11

Munoz-Quezada, S., Bermudez-Brito, M., Chenoll, E., Genoves, S., GomezLlorente, C., Plaza-Diaz, J., et al. (2013). Competitive inhibition of three novel bacteria isolated from faeces of breast milk-fed infants against selected enteropathogens. Br. J. Nutr. 109(Suppl. 2), S63-S69. doi: 10.1017/ S0007114512005600 
Parkes, G. C., Sanderson, J. D., and Whelan, K. (2009). The mechanisms and efficacy of probiotics in the prevention of Clostridium difficile-associated diarrhoea. Lancet Infect. Dis. 9, 237-244. doi: 10.1016/S1473-3099(09) 70059-3

Petrosillo, N. (2018). Tackling the recurrence of Clostridium difficile infection. Med. Mal. Infect. 48, 18-22. doi: 10.1016/j.medmal.2017.10.007

Pothoulakis, C., Kelly, C. P., Joshi, M. A., Gao, N., O’Keane, C. J., Castagliuolo, I., et al. (1993). Saccharomyces boulardii inhibits Clostridium difficile toxin A binding and enterotoxicity in rat ileum. Gastroenterology 104, 1108-1115. doi: 10.1016/0016-5085(93)90280-P

Ratsep, M., Koljalg, S., Sepp, E., Smidt, I., Truusalu, K., Songisepp, E., et al. (2017). A combination of the probiotic and prebiotic product can prevent the germination of Clostridium difficile spores and infection. Anaerobe 47, 94-103. doi: 10.1016/j.anaerobe.2017.03.019

Ripert, G., Racedo, S. M., Elie, A. M., Jacquot, C., Bressollier, P., and Urdaci, M. C. (2016). Secreted compounds of the probiotic Bacillus clausii strain $\mathrm{O} / \mathrm{C}$ inhibit the cytotoxic effects induced by Clostridium difficile and Bacillus cereus toxins. Antimicrob. Agents Chemother. 60, 3445-3454. doi: 10.1128/AAC. 02815-15

Rolfe, R. D. (2000). The role of probiotic cultures in the control of gastrointestinal health. J. Nutr. 130(2S Suppl.), 396S-402S. doi: 10.1093/jn/130.2.396S

Rupnik, M., Wilcox, M. H., and Gerding, D. N. (2009). Clostridium difficile infection: new developments in epidemiology and pathogenesis. Nat. Rev. Microbiol. 7, 526-536. doi: 10.1038/nrmicro2164

Schoster, A., Kokotovic, B., Permin, A., Pedersen, P. D., Dal Bello, F., and Guardabassi, L. (2013). In vitro inhibition of Clostridium difficile and Clostridium perfringens by commercial probiotic strains. Anaerobe 20, 36-41. doi: 10.1016/j.anaerobe.2013.02.006

Sheu, S. J., Hwang, W. Z., Chiang, Y. C., Lin, W. H., Chen, H. C., and Tsen, H. Y. (2010). Use of tuf gene-based primers for the PCR detection of probiotic Bifidobacterium species and enumeration of bifidobacteria in fermented milk by cultural and quantitative real-time PCR methods. J. Food Sci. 75, M521-M527. doi: 10.1111/j.1750-3841.2010.01816.x

Spinler, J. K., Auchtung, J., Brown, A., Boonma, P., Oezguen, N., Ross, C. L., et al. (2017). Next-generation probiotics targeting Clostridium difficile through precursor-directed antimicrobial biosynthesis. Infect. Immun. 85:e00303-17. doi: 10.1128/IAI.00303-17

Trejo, F. M., De Antoni, G. L., and Perez, P. F. (2013). Protective effect of bifidobacteria in an experimental model of Clostridium difficile associated colitis. J. Dairy Res. 80, 263-269. doi: 10.1017/S0022029913000216
Trejo, F. M., Perez, P. F., and De Antoni, G. L. (2010). Co-culture with potentially probiotic microorganisms antagonises virulence factors of clostridium difficile in vitro. Antonie Van Leeuwenhoek 98, 19-29. doi: 10.1007/s10482-010-9424-6

Valdes-Varela, L., Alonso-Guervos, M., Garcia-Suarez, O., Gueimonde, M., and Ruas-Madiedo, P. (2016). Screening of Bifidobacteria and Lactobacilli able to antagonize the cytotoxic effect of Clostridium difficile upon intestinal epithelial HT29 monolayer. Front. Microbiol. 7:577. doi: 10.3389/fmicb.2016.00577

Vemuri, R. C., Gundamaraju, R., Shinde, T., and Eri, R. (2017). Therapeutic interventions for gut dysbiosis and related disorders in the elderly: antibiotics, probiotics or faecal microbiota transplantation? Benef. Microbes 8, 179-192. doi: 10.3920/BM2016.0115

Wei, Y., Li, Y., Yang, F., Wu, Q., Liu, D., Li, X., et al. (2017). Physical and functional interplay between MazF1Bif and its noncognate antitoxins from Bifidobacterium longum. Appl. Environ. Microbiol. 83:e03232-16. doi: 10.1128/ AEM.03232-16

Wei, Y. X., Zhang, Z. Y., Liu, C., Zhu, Y. Z., Zhu, Y. Q., Zheng, H., et al. (2010). Complete genome sequence of Bifidobacterium longum JDM301. J. Bacteriol. 192, 4076-4077. doi: 10.1128/JB.00538-10

Xu, H., Jeong, H. S., Lee, H. Y., and Ahn, J. (2009). Assessment of cell surface properties and adhesion potential of selected probiotic strains. Lett. Appl. Microbiol. 49, 434-442. doi: 10.1111/j.1472-765X.2009.02684.x

Yang, J., Zhang, H., Jiang, L., Guo, H., Luo, X., and Ren, F. (2015). Bifidobacterium longum BBMN68-specific modulated dendritic cells alleviate allergic responses to bovine beta-lactoglobulin in mice. J. Appl. Microbiol. 119, 1127-1137. doi: $10.1111 /$ jam. 12923

Yun, B., Song, M., Park, D. J., and Oh, S. (2017). Beneficial effect of Bifidobacterium longum ATCC 15707 on survival rate of Clostridium difficile infection in mice. Korean J. Food Sci. Anim. Resour. 37, 368-375. doi: 10.5851/kosfa.2017.37.3.368

Conflict of Interest Statement: The authors declare that the research was conducted in the absence of any commercial or financial relationships that could be construed as a potential conflict of interest.

Copyright (C) 2018 Wei, Yang, Wu, Gao, Liu, Liu, Guo, Suwal, Kou, Zhang, Wang, Zheng and Tang. This is an open-access article distributed under the terms of the Creative Commons Attribution License (CC BY). The use, distribution or reproduction in other forums is permitted, provided the original author(s) and the copyright owner are credited and that the original publication in this journal is cited, in accordance with accepted academic practice. No use, distribution or reproduction is permitted which does not comply with these terms. 